\title{
Eficacia de los hipoglicemiantes orales en el control metabólico de pacientes con diabetes mellitus gestacional
}

\author{
Enrique Valdés $R^{1}$, Emiliano Soto-Chacón ${ }^{1,2}$, \\ Rodolfo Lahsen $\mathrm{M}^{3}$, Carlos Barrera $\mathrm{H}^{4}$, Paula Candia $\mathrm{P}^{4}$. \\ Effectiveness of oral hypoglycemic \\ drugs in the metabolic control of \\ patients with gestational diabetes
}

Gestational Diabetes is characterized by different degrees of glucose intolerance that produce a series of fetal and perinatal alterations. During many years, in those cases of gestational diabetes that did not respond to nutritional interventions, the use of insulin was a proven treatment to achieve metabolic control and thus a better perinatal outcome. At present, some new oral hypoglycemic drugs, from the family of sulfonylureas and biguanides, have been shown to be safe, of low cost, and apparently effective in the metabolic control of this disease. We review the publications that propose the use of oral hypoglycemic drugs for the metabolic control of gestational diabetes that does not respond to nutritional measures (Rev Méd Chile 2008; 136: 915-20).

(Key w ords: Diabetes, gestational; Hypoglycemic agents; Insulin)

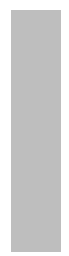

Recibido el 2 de abril, 2007. Aceptado el 27 de agosto, 2007.

${ }^{1}$ Unidad de Medicina Materno Fetal, Departamento de Obstetricia y Ginecología, Hospital Clínico Universidad de Chile. ${ }^{2}$ Programa Doctorado en Ciencias Médicas y Formación de Especialista en Obstetricia y Ginecología, Facultad de Medicina, Universidad de Chile. ${ }^{3}$ Departamento de Medicina, Sección Endocrinología, Hospital Clínico Universidad de Chile. ${ }^{4}$ Unidad Medicina Materno Fetal, Servicio de Obstetricia y Ginecología, Hospital Barros Luco-Trudeau. Santiago de Chile.

L a diabetes mellitus gestacional (DMG) es una patología caracterizada por una alteración del metabolismo de los hidratos de carbono de severidad variable que se inicia o pesquisa por primera vez durante el embarazo. En Chile pre-

Correspondencia a: Dr. Enrique Valdés Rubio. Departamento de Obstetricia y Ginecología. Hospital Clínico Universidad de Chile. Santos Dumont 999, Independencia. E mail: evaldes@vtr.net senta una prevalencia en la población general de embarazadas de $3 \%$ a $5 \%$, llegando al $10 \%$ a $14 \%$, si consideráramos a gestantes con factores de riesgo diabetógeno. Sin embargo, es una patología que debe pesquisarse en toda embarazada, ya que aproximadamente $50 \%$ de los casos de DMG no presentan estos factores de riesgo ${ }^{1,2}$.

Se manifiesta en el segundo o tercer trimestre del embarazo, diagnosticándose con una prueba de tolerancia oral a glucosa (PTGO) alterada o dos glicemias de ayuno elevadas. Pilares diagnósticos 
sugeridos en la Cuarta Jornada Internacional de Grupo de Trabajo en Diabetes Gestacional, adoptados por el Colegio Americano de Obstetricia y Ginecología y basados en los criterios de Carpenter y Constan ${ }^{3}$.

En esta misma enfermedad se distinguen dos formas de presentación o severidad:

1. Intolerancia a hidratos de carbono que presenta glicemias $2 \mathrm{~h}$ postcarga entre 140 y 199 $\mathrm{mg} / \mathrm{dl}$.

2. Diabetes gestacional propiamente tal con glicemias a las $2 \mathrm{~h}$ postcarga mayor de 200 $\mathrm{mg} / \mathrm{dl}$.

A pesar de ser de mejor pronóstico que otras formas de diabetes mellitus, la DMG se asocia con malos resultados materno-fetales, observándose por una parte un aumento de las complicaciones maternas y por otra, una mayor incidencia de inmadurez, macrosomía fetal e hipoglicemia neonatal, condiciones precipitantes del aumento de las tasas de morbimortalidad perinatal observada en hijos de mujeres embarazadas con un inadecuado control metabólico ${ }^{4,5}$.

Siendo que la glicemia fetal está determinada en forma directa por la glicemia materna, alteraciones en este parámetro materno condicionarán cambios en el metabolismo fetal, apareciendo hiperinsulinemia en el producto6,7. No sólo el manejo de los hidratos de carbono se altera, también se producen incrementos fluctuantes en el plasma fetal de ácidos grasos libres, triglicéridos, cuerpos cetónicos y algunos aminoácidos ${ }^{8}$. Todas estas alteraciones son más acentuadas en embarazadas con mal control metabólico.

Por otra parte, el recién nacido está expuesto a una serie de alteraciones metabólicas secundarias a un deficitario control metabólico materno-fetal (hipoglicemia, hipocalcemia, policitemia, hiperbilimubinemia y disminución de la síntesis de lecitina) que le impiden una buena adaptación a su vida extrauterina, aumentando su morbimortalidad neonatal, siendo esta condición más frecuente en hijos de madres diabéticas con mayor descompensación metabólica9-11.

Por último, gran parte de los efectos deletéreos que se observan en la DMG se deben a un mal manejo metabólico de ella, en donde la paciente persiste con hiperglicemias mantenidas. Es por esto que el pilar terapéutico fundamental de esta patología es el régimen dietético y en los casos refractarios el uso de insulinoterapia, que si bien es cierto es efectiva, presenta todas las desventajas que conlleva un tratamiento inyectable, de alto costo y de uso complejo en forma ambulatoria.

La dietoterapia se administra a todas las mujeres con una prueba de sobrecarga a 75 gramos de glucosa a los 120 min (PTGO) alterada, con el objetivo metabólico de lograr glicemias en ayunas entre 70 y $90 \mathrm{mg} / \mathrm{dl}$, glicemias de $2 \mathrm{~h}$ postcarga entre 90 y $120 \mathrm{mg} / \mathrm{dl}$, y ausencia de glucosuria y cetonuria.

Junto con la dieta, es necesario estimular la actividad física y el autocuidado de la paciente.

En aquellos casos en que no se logre un adecuado manejo metabólico a pesar de las medidas antes descritas, la insulinoterapia es el tratamiento aconsejable. Esta terapia en muchos casos debe iniciarse hospitalizando a la paciente, de modo de monitorizar la aparición de síntomas de hipoglicemia, requiriendo que la paciente sea educada en su forma y vía de administración. Todo esto conlleva el comprensible rechazo que produce este tratamiento en algunas mujeres, no sólo por su forma de administración (parenteral) sino que también por su elevado costo monetario.

Por otra parte, el uso de hipoglicemiantes orales (HGO) en el tratamiento de la DMG refractaria a la dieta, ha sido históricamente rechazado por la comunidad médica, sobre la base de unos pocos estudios de tipo observacional con serias falencias metodológicas ${ }^{12-14}$.

En Estados Unidos de Norteamérica (EE.UU.), el uso de HGO en el tratamiento de la DMG está contraindicado, sobre la base de tres premisas que hoy en día no tienen asidero científico:

1. Mayor frecuencia de malformaciones congénitas.

2. Probable inducción de macrosomía fetal, secundaria a la estimulación directa del páncreas fetal con la consecuente hiperinsulinemia.

3. Mayor frecuencia de hipoglicemias neonatales secundarias a hiperinsulinemia fetal.

\section{SULFONILUREAS}

Las sulfonilureas se han caracterizado por ser las principales drogas hipoglicemiantes utilizadas en el control de la diabetes mellitus tipo 2. Las de 
primera generación incluyen entre otras tolazamida, clorpropamida y tolbutamida. En los últimos años, se han desarrollado las sulfonilureas de segunda generación, donde se destacan glibenclamida, glipizida y glimepirida. La acción farmacológica de estas se basa en la liberación de insulina por parte de las células beta pancreáticas $\mathrm{y}$, secundariamente, aumentando la sensibilidad tisular periférica de la insulina ${ }^{15,16}$.

Actualmente existe suficiente información que avalaría que las sulfonilureas de segunda generación como la glibenclamida son seguros durante el embarazo (FDA- B) y han demostrado ser tan eficaces como la insulinoterapia en el control de la hiperglicemia de las gestantes refractarias a la restricción calórica ${ }^{17-19}$. En este sentido, en los últimos cinco años han aparecido en la literatura estudios que han derribado varios de los prejuicios que se tenían frente al uso de $\mathrm{HGO}$ en el tratamiento de la DMG. Es así como estudios sobre el paso transplacentario de glibenclamida, demostraron que insignificantes cantidades de este fármaco podían atravesar la barrera placentaria $^{20}$ conduciendo a otras importantes conclusiones:

1. Los niveles plasmáticos del fármaco, así como la concentración plasmática de albúmina, no afectan la velocidad de transferencia del fármaco hacia el feto.

2. La glibenclamida no es secuestrada ni metabolizada por la placenta.

3. La tasa de intercambio feto-madre es similar a la tasa de intercambio madre-feto.

Estas conclusiones fueron avaladas por el hecho de que no se pudo detectar glibenclamida en sangre de cordón umbilical en recién nacidos de madres con DMG en tratamiento con este fármaco ${ }^{17}$. Estas conclusiones también son válidas en placentas de madres con DMG, observándose el mismo patrón antes descrito 21,22 .

La razón de por qué la glibenclamida no cruzaría la barrera placentaria fue postulada por Koren, quien sugiere un modelo en el cual la glibenclamida no cruza esta barrera porque presenta una alta unión a proteínas plasmáticas como la albúmina (99,8\%), siendo esta característica constante aun en situaciones experimentales en las que los niveles plasmáticos del fármaco superan 10 veces el nivel terapéutico. Por otra parte, la vida media reducida de este fármaco $(4 \mathrm{~h})$ y su rápida eliminación $(1,3 \pm 0,5 \mathrm{~mL} / \mathrm{kg} / \mathrm{min})$ también juegan a favor de su escaso paso transplacentario ${ }^{23}$.

Hasta la fecha se han reportado sólo 2 estudios clínicos con muestra aleatoria que han evaluado el uso de sulfonilureas durante el embarazo ${ }^{17-19}$, no existiendo evidencias publicadas a nivel nacional. Notelowitz indagó sobre la eficacia de la tolbutamida, clorpropamida, dieta e insulina en el manejo de la diabetes pregestacional, en un modelo aleatorio. Este estudio no encontró diferencias significativas en cuanto a mortalidad neonatal y tasa de malformaciones congénitas entre los grupos $^{15}$.

El segundo estudio incluyó a 404 mujeres que fueron distribuidas en forma aleatoria entre las 11 y 33 semanas de embarazo, para comparar la eficacia de la glibenclamida y la insulina en el manejo de la DMG. Aquí, 82\% de las mujeres tratadas con glibenclamida y $88 \%$ de las mujeres tratadas con insulina lograron un control metabólico satisfactorio, definido como promedio de glicemias entre 90 y $105 \mathrm{mg} / \mathrm{ml}$. Sólo ocho mujeres (4\%) en tratamiento con glibenclamida no lograron un buen manejo metabólico, necesitando el uso de insulina, aun con dosis máxima de este fármaco (20 mg/día). Sin embargo, la ocurrencia de hipoglicemias $(<40 \mathrm{mg} / \mathrm{dl})$ fue más frecuente en el grupo tratado con insulina $(41 \mathrm{v} / \mathrm{s} 4 ; \mathrm{p}=$ $0,03)$. Entre estos grupos no se encontraron diferencias significativas en cuanto a resultado perinatal, vía de parto, incidencia de preeclampsia, frecuencia de macrosomía y mortalidad neonatal ${ }^{17}$.

Recientemente, un estudio reafirma la seguridad y efectividad de la glibenclamida, concluyendo que $81 \%$ (IC 95\%; 76,4-85,6) de las diabéticas gestacionales tratadas con este fármaco alcanzaron un adecuado control metabólico sin presentar riesgos teratogénicos ni metabólicos en el neonato, con sólo $1 \%$ de abandono de la terapia por efectos colaterales. Sin embargo, 19\% de los hijos de las madres tratadas presentaron macrosomía al nacer ${ }^{18}$.

Por otra parte, un estudio posterior retrospectivo de un total de 504 pacientes refractarias a la dietoterapia, donde 236 recibieron glibenclamida y 268 insulina, concluyó que la glibenclamida sería más efectiva en el logro de las metas 
metabólicas que la insulinoterapia ( $86 \%$ v/s 63\%; $p<0,001)$, sin embargo, encontraron en el grupo tratado con glibenclamida una mayor incidencia de preeclampsia $(12 \% \mathrm{v} / \mathrm{s} 6 \% ; \mathrm{p}=0,02)$, una mayor necesidad de fototerapia en los hijos de aquellas madres tratadas con este fármaco $(9 \% \mathrm{v} / \mathrm{s}$ $5 \% ; \mathrm{p}<0,05)$ y sin diferencias significativas del peso al nacer entre ambos grupos, es importante destacar que este trabajo presenta algunos sesgos metodológicos, al ser un estudio retrospectivo ${ }^{19}$, por lo que algunas de estas conclusiones deben ser confirmadas en un ensayo clínico prospectivo y aleatorio.

\section{BigUANIDAS}

Las biguanidas ejercen su acción principalmente mediante dos mecanismos, el primero, disminuyendo los niveles séricos de glucosa a través de la reducción de la gluconeogénesis hepática y el segundo, sensibilizando los tejidos periféricos a la acción de la insulina ${ }^{24}$.

Mientras que la biguanida de primera generación, fenformina, fue retirada del mercado por asociarse con acidosis láctica, la metformina, una biguanida de segunda generación, demostró ser bastante más segura, ya que la incidencia de acidosis encontrada fue sólo de 0,03/1.000 pacientes/año, por lo que la Administración de Drogas y Alimentos de los Estados Unidos de Norteamérica (FDA) la aprobó para su uso en categoría $\mathrm{B}^{25}$.

La metformina ha comprobado ser de utilidad en el tratamiento de una serie de patologías como por ejemplo el síndrome del ovario poliquístico (SOP), la infertilidad de causa ovulatoria y en el aborto de primer trimestre ${ }^{26}$. Por otra parte, el uso de la metformina ha demostrado ser seguro durante el embarazo, a pesar que cruza libremente la placenta alcanzando en el feto concentraciones plasmáticas similares a las maternas ${ }^{27}$, ya que no ha demostrado tener un efecto importante en el transporte de glucosa fetal, siendo el consumo de glucosa a nivel placentario mínimo, no presentando efectos teratogénicos y no provocando hipoglicemia neonatales, por lo que su uso en diabetes gestacional parece adecuado. En relación con esto último, existen sólo algunos estudios retrospectivos que indican resultados controverti- dos, uno de ellos refiere que su uso durante la segunda mitad del embarazo estaría asociado a un incremento de la prevalencia de preeclampsia y una alta mortalidad perinatal ${ }^{28}$. Más recientemente, Hughes concluye, en una muestra de 214 pacientes, que las diabéticas gestacionales tratadas con metformina versus los controles no presentaron diferencias significativas en el riesgo de presentar mal resultado perinatal ${ }^{29}$. Controversias que al parecer se aclararon parcialmente con la publicación del estudio multicéntrico neocelandés y australiano donde se concluye que la metformina sola o suplementada con insulina no está asociada a un aumento de las complicaciones perinatales comparada con el uso de la insulina, pospone la indicación de insulinoterapia, disminuye la dosis de insulina diaria indicada y presenta preferencia por parte de las gestantes al momento de elegir una u otra terapia ${ }^{30}$, esta experiencia avaló al National Institute for Health and Clinical Excellence a sugerir el uso de metformina como tratamiento alternativo o complementario a la insulinoterapia ${ }^{31}$.

Por otra parte, en los países desarrollados, especialmente EE.UU., existen experiencias en las cuales se ha demostrado un ahorro significativo al utilizar glibenclamida v/s insulinoterapia, ahorro que asciende a un promedio de US\$165 por paciente durante el embarazo ${ }^{32}$. En nuestro país, los estudios de costo efectividad se han ido desarrollando de manera paulatina, incorporando desde hace pocos años la valoración monetaria de las acciones clínicas bajo un enfoque complementario.

En conclusión, basado en que se ha demostrado que la DMG y la diabetes mellitus tipo 2 comparten una fisiopatología similar, con aumento de la resistencia a la insulina, mal control de la gluconeogénesis hepática y una respuesta de células beta pancreática disminuida ${ }^{33}$, es posible plantear que glibenclamida o metformina serían una terapia segura, efectiva, de bajo costo y de fácil uso ambulatorio en gestantes con DMG que no respondan a régimen dietético, necesitándose aún estudios clínicos metodológicamente bien estructurados que respondan definitivamente el rol que juegan estos hipoglicemiantes en el control metabólico de las pacientes diabéticas gestacionales que no responden a régimen nutricional. 


\section{REFERENCIAS}

1. Sepe SJ, Connell FA, Geiss LS, Teutsch SM. Gestational diabetes. Incidence, maternal characteristics, and perinatal outcome. Diabetes 1985; 34 Suppl 2: 13-6.

2. Landon MB, Gabbe SG. Diabetes and pregnancy. Med Clin North Am. 1988; 72: 1493-511.

3. ACOG Practice Bulletin. Clinical management guidelines for obstetrician-gynecologists. Gestational diabetes. Obstet Gynecol 2001; 98: 525-38.

4. Gabbe SG. Gestational diabetes mellitus. N Engl J Med 1986; 315: 1025-6.

5. Kühl C, Moldsted-Pedersen L. Pregnancy and diabetes: gestational diabetes. Diabetes Annu 1987; 3: 242-51.

6. Adam PA, Teramo K, Raiha N, Gitun D, Schwartz R. Human fetal insulin metabolism early in gestation. Response to acute elevation of the fetal glucose concentration and placental transfer of human insulin-I-131. Diabetes. 1969; 18: 409-16.

7. Milner RD, Ashworth MA. Barson AJ. Insulin release from human foetal pancreas in response to glucose, leucine and arginine. J Endocrinol. 1972; 52: 497-505.

8. Pedersen J. The pregnant diabetic and her newborn: Problems and management. Filadelfia: Editorial Williams \& Wilkins, 1967; 113-26.

9. Gillmer MD, Beard RW, Brooke FM, OAKley NW. Carbohydrate metabolism in pregnancy. Part I. Diurnal plasma glucose profile in normal and diabetic women. BMJ 1975; 3: 399-402.

10. Andersen O, Hertel J, Schmolker L, Kuhl C. Influence of the maternal plasma glucose concentration at delivery on the risk of hypoglycaemia in infants of insulin-dependent diabetic mothers. Acta Paediatr Scand 1985; 74: 268-73.

11. Moya FY, Gross I. Diabetes and fetal lung development. En: Reece EA, Cosutand DR eds. Diabetes Mellitus in Pregnancy: Principles and Practice. Londres: Editorial Churchill Livingstone 1988; 123-38.

12. Zucker P, Simon G. Prolonged symptomatic neonatal hypoglycemia associated with maternal chlorpropamide therapy. Pediatrics 1968; 42: 824-5.

13. Kemball ML, McIver C, Milner RD, Nourse CH, SCHIFF D, Tiernan JR. Neonatal hypoglycaemia in infants of diabetic mothers given sulphonylurea drugs in pregnancy. Arch Dis Child 1970; 45: 696-701.

14. Piacquadio K, Howingsworth DR, Murphy H. Effects of in-utero exposure to oral hypoglycaemic drugs. Lancet 1991; 338: 866-9.

15. Notelovitz M. Sulphonylurea therapy in the treatment of the pregnant diabetic. S Afr Med J 1971; 45: 226-9.
16. Groop L, Luzi L, Melander A, Groop PH, Ratheiser K, Simonson DC, DeFronzo RA. Different effects of glyburide and glipizide on insulin secretion and hepatic glucose production in normal and NIDDM subjects. Diabetes 1987; 36: 1320-8.

17. Langer O, Conway DL, Berkus MD, Xenakis EM, GonZales O. A comparison of glyburide and insulin in women with gestational diabetes mellitus. N Engl J Med 2000; 343: 1134-8.

18. KREMER CJ, DufF P. Glyburide for the treatment of gestational diabetes. Am J Obstet Gynecol 2004; 190: 1438-9.

19. Jacobson GF, Ramos GA, Ching JY, Kirby RS, Ferrara A, FIELD DR. Comparison of glyburide and insulin for the management of gestational diabetes in a lange managed care organization. Am J Obstet Gynecol 2005; 193: 118-24.

20. ELLott BD, Langer O, Schenker S, Johnson RF. Insignificant transfer of glyburide occurs across the human placenta. Am J Obstet Gynecol 1991; 165: 807-12.

21. Eluitt BD, Schenker S, Langer O, Johnson R, PRIHODA T. Comparative placental transport of oral hypoglycemic agents in humans: a model of human placental drug transfer. Am J Obstet Gynecol 1994; 171: 653-60.

22. Elitott BD, Langer O, Schuessling F. Human placental glucose uptake and transport are not altered by the oral antihyperglycemic agent metformin. Am J Obstet Gynecol 1997; 176: 52730.

23. Koren G. Glyburide and fetal safety; transplacental pharmacokinetic considerations. Reprod Toxicol 2001; 15: 227-9.

24. Sirtori CR, PASIK C. Re-evaluation of a biguanide, metformin: mechanism of action and tolerability. Pharmacol Res 1994; 30: 187-228.

25. Briggs GG, Yaffe SJ, FreEman RK. Drugs in Pregnancy and Lactation: A Reference Guide to Fetal and Neonatal Risk. Filadelfia. Editorial Lippincott Williams \& Wilkins, 2005; 687-90.

26. McCarthy EA, Walker SP, McLachlan K, Boyle J, Permezel M. Metformin in Obstetric and Gynecologic Practice: A Review. Obstet Gynecol Surv 2004; 59: 118-27.

27. Vanky E, Zahlsen K, Spigset O, Carlsen SM. Placental passage of metformin in women with polycystic ovary syndrome. Fertil Steril 2005; 83: 1575-8.

28. Hellmuth E, Damm P, Molsted-Pedersen L. Oral hypoglycaemic agents in 118 diabetic pregnancies. Diabet Med 2000; 17: 507-11.

29. Hughes RC, Rowan JA. Pregnancy in women with Type 2 diabetes: who takes metformin and what is the outcome? Diabet Med 2006; 23: 318-22.

30. Rowan JA, Hague W, Gao W, Battin M, Moore P. 
Metformin versus Insulin for the treatment of gestational diabetes. N Engl J Med 2008; 358: 2003-15.

31. National Institute for Health and Clinical Excellence. Guías en Diabetes y Embarazo www.nice.org.uk/guidance/index.jsp?action- =byID \&0 =11946. Consultado en junio 2008.
32. GoEtzL L, WiLKINS I. Gliburide compared to insulin for the treatment of gestational diabetes mellitus. A cost analysis. J Perinat 2002; 22: 403-6.

33. American Diabetes Association. Implications of the United Kingdom Prospective Diabetes Study. Diabetes Care 2000; 23 Suppl 1: S27-31. 